OPEN ACCESS

Edited by:

Randy D. Allen,

Oklahoma State University,

United States

Reviewed by:

Trevor M. Nolan,

Duke University, United States

Xingyun Qi,

Rutgers, The State University

of New Jersey, United States

*Correspondence:

Nobutoshi Yamaguchi

nobuy@bs.naist.jp

Toshiro Ito

itot@bs.naist.jp

Specialty section:

This article was submitted to

Plant Abiotic Stress,

a section of the journal

Frontiers in Plant Science

Received: 24 August 2020 Accepted: 03 November 2020

Published: 25 November 2020

Citation:

Wu J, Yan $M$, Zhang $D$, Zhou $D$, Yamaguchi $N$ and lto $T$ (2020) Histone

Demethylases Coordinate the Antagonistic Interaction Between Abscisic Acid and Brassinosteroid

Signaling in Arabidopsis.

Front. Plant Sci. 11:596835.

doi: 10.3389/fpls.2020.596835

\section{Histone Demethylases Coordinate the Antagonistic Interaction Between Abscisic Acid and Brassinosteroid Signaling in Arabidopsis}

\author{
Jinfeng Wu $\mathbf{u}^{1,2,3}$, Mingli Yan ${ }^{1,3}$, Dawei Zhang 1,3, Dinggang Zhou ${ }^{1,3}$, \\ Nobutoshi Yamaguchi ${ }^{2,4 *}$ and Toshiro Ito ${ }^{2 *}$
}

${ }^{1}$ School of Life Sciences, Hunan University of Science and Technology, Xiangtan, China, ${ }^{2}$ Division of Biological Science, Nara Institute of Science and Technology, Ikoma, Japan, ${ }^{3}$ Hunan Key Laboratory of Economic Crops Genetic Improvement and Integrated Utilization, Hunan University of Science and Technology, Xiangtan, China, ${ }^{4}$ Precursory Research

for Embryonic Science and Technology, Japan Science and Technology Agency, Kawaguchi-shi, Japan

Abscisic acid (ABA) interacts antagonistically with brassinosteroids (BRs) to control plant growth and development in response to stress. The response to environmental cues includes hormonal control via epigenetic regulation of gene expression. However, the details of the ABA-BR crosstalk remain largely unknown. Here, we show that JUMONJI$\mathrm{C}$ domain containing histone demethylases (JMJs) coordinate the antagonistic interaction between $\mathrm{ABA}$ and $\mathrm{BR}$ signaling pathways during the post-germination stage in Arabidopsis. BR blocks ABA-mediated seedling arrest through repression of JMJ30. JMJs remove the repressive histone marks from the BRASSINAZOLE RESISTANT1 (BZR1) locus for its activation to balance ABA and BR signaling pathways. JMJs and BZR1 co-regulate genes encoding three membrane proteins, a regulator of vacuole morphology, and two lipid-transfer proteins, each of which play a different role in transport. BZR1 also regulates stimuli-related target genes in a JMJ-independent pathway. Our findings suggest that the histone demethylases integrate ABA and BR signals, leading to changes in growth program after germination.

\footnotetext{
Keywords: abscisic Acid, Arabidopsis thaliana, BRASSINAZOLE RESISTANT1, brassinosteroids, JUMONJI C demethylases
}

\section{INTRODUCTION}

Plants have the ability to respond to various environmental stimuli over the course of their development (Blatt et al., 2017; Bechtold and Field, 2018). These responses are characterized by extensive phenotypic plasticity, and entail a balance between stress response and growth (Foyer et al., 2007; Sanchez-Bel et al., 2018). In this flexible and reversible balance, plants minimize the damage caused by stress and maximize growth. However, we do not fully understand how plants control this intricate balance.

The phytohormone abscisic acid (ABA) coordinates stress responses and inhibits plant growth (Yamaguchi-Shinozaki and Shinozaki, 2006; Cutler et al., 2010; Finkelstein, 2013). In the absence of $\mathrm{ABA}$, type $2 \mathrm{C}$ protein phosphatases (PP2Cs) physically interact with SNF1-RELATED PROTEIN KINASE 2s (SnRK2s) to inhibit protein phosphorylation (Hrabak et al., 2003). ABA binds to 
receptors such as PYRABACTIN RESISTANCE1 (PYR1), PYR1LIKE (PYL), and REGULATORY COMPONENTS OF ABA RECEPTOR (RCAR). This triggers conformational changes that enable protein-protein interaction between PYRs and PP2Cs, such as ABSCISIC ACID INSENSITIVE 1 (ABI1), ABI2, and HYPERSENSITIVE TO ABA1 (HAB1; Park et al., 2009; Cutler et al., 2010). The conformational changes release SnRK2s from inhibition by PP2Cs. The SnRK2s activate downstream transcription factors such as the bZIP-type transcription factor ABSCISIC ACID INSENSITIVE5 (ABI5), the AP2-type protein $\mathrm{ABI} 4$, the B3-type protein $\mathrm{ABI} 3$, and ABA-responsive elementsbinding factors (ABFs), through phosphorylation (Lopez-Molina et al., 2002; Nakashima et al., 2009). These transcription factors regulate the expression of $\mathrm{ABA}$-responsive genes including stress response and growth-inhibition targets (Suzuki et al., 2003; Nakabayashi et al., 2005).

Another class of phytohormones, BRs, promote plant growth and development. The plasma membrane-localized serine/threonine kinase BRASSINOSTEROID-INSENSITIVE1 (BRI1) recognizes BRs (Clouse, 2011; Zhang et al., 2018). BRI1-BR binding triggers conformational changes that enable heterodimer formation between BRI1 and BRASSINOSTEROID INSENSITIVE-ASSOCIATED RECEPTOR KINASE 1 (BAK1; Li and Chory, 1997; Nam and Li, 2002; Cano-Delgado et al., 2004; Fabregas et al., 2013). These changes initiate intracellular phosphorylation pathways: the BRI1/BAK1 heterodimer leads to promotion of the activity and stability of the plantspecific transcription factors BRASSINAZOLE RESISTANT1 (BZR1) and bri1-EMS-SUPPRESSOR1 (BES1), which lead to transcriptional activation of BR-responsive genes for plant growth and development (Yin et al., 2002; Ibanez et al., 2018; Tian et al., 2018).

Recent studies suggest that ABA and BRs antagonistically regulate environmental responses ( $\mathrm{Hu}$ and $\mathrm{Yu}, 2014$; Ryu et al., 2014; Skubacz et al., 2016). Brassinosteroid biosynthetic and signaling mutants are hypersensitive to exogenous ABA; they are hyposensitive to ABA when these BR biosynthetic and signaling genes are overexpressed (Clouse et al., 1996). BIN2 is a key module that controls BR-ABA crosstalk (Gruszka, 2013). BIN2 stabilizes ABI5 through phosphorylation in the presence of ABA (Hu and Yu, 2014). SnRK2.2, SnRK2.3, and SnRK2.6 are also phosphorylation substrates of BIN2 (Cai et al., 2014; Shang et al., 2016). In addition, BZR1 directly regulates ABI5 expression (Yang et al., 2016). Hence, the transcriptional regulatory network underlying the ABA-BR crosstalk is at least partially characterized.

Epigenetic regulation mediates reversible changes in gene expression. The type and degree of histone modification is often affected by environmental stimuli (Kim et al., 2008; Luo et al., 2012). H3 lysine 27 trimethylation (H3K27me3) is associated with a repressed chromatin state and gene silencing (Zhang et al., 2007; Lafos et al., 2011; Wu et al., 2012; Sun et al., 2014), and is responsive to environmental changes (Liu et al., 2014; Qian et al., 2018). H3 lysine 27 trimethylation demethylases have important roles in regulating these dynamics (Tsukada et al., 2006; Yamane et al., 2006). EARLY FLOWERING 6 (ELF6), RELATIVE OF EARLY FLOWERING 6 (REF6), JUMONJI C
DOMAIN-CONTAINING PROTEIN 13 (JMJ13), JMJ30, and JMJ32 function as $\mathrm{H} 3 \mathrm{~K} 27 \mathrm{me} 3$ demethylases in Arabidopsis (Lu et al., 2011; Gan et al., 2014; Zheng et al., 2019). In response to ABA, ABI3-mediated JMJ30 activation accelerates SnRK2.8 expression through $\mathrm{H} 3 \mathrm{~K} 27 \mathrm{me} 3$ demethylation (Wu et al., 2019a,b). ELF6, REF6, and BES1 form a complex in response to BRs, and control genes downstream of BES1 through H3 lysine9 trimethylation (H3K9me3; Yu et al., 2008). However, the role of $\mathrm{H} 3 \mathrm{~K} 27 \mathrm{me} 3$ demethylases on the crosstalk between $\mathrm{ABA}$ and BR signaling pathways remains unclear.

Here, we provide insights into the role of histone demethylases in the antagonistic interaction between ABA and BRs during post-germination stage. BR blocks ABA-mediated seedling arrest through JMJ30 repression. JMJ30 and JMJ32 remove H3K27me3 marks from the BZR1 locus to activate BZR1 expression. Finally, we identified genes that were shared and unique targets of JMJ and BZR1; these were involved in transport and stress responses. Our study reveals a shared regulatory network between ABA and BR that acts via JMJ-mediated histone modification and a BR-signaling master regulator.

\section{MATERIALS AND METHODS}

\section{Plant Materials and Plant Growth}

In this study, the Arabidopsis thaliana Columbia (Col-0) was used and the mutants are in the Col-0 background. The jmj30-2 jmj321 mutant, pJMJ30:JMJ30-GUS, and PJMJ30:JMJ30-HA have been described previously (Gan et al., 2014). Seeds were stratified for 3 days at $4^{\circ} \mathrm{C}$ in darkness. The plants were grown in a growth chamber at $22^{\circ} \mathrm{C}$ under continuous light.

\section{Plasmid Construction and Plant Transformation}

To generate a BZR1 overexpression construct, the full-length coding sequences (CDS) were inserted into the pENTR/D-TOPO vector (Thermo Fisher Scientific) using the primers, BZR1cds_FW and BZR1-cds_RV. Then, the BZR1 CDS-containing vector was recombined into pB2GW7.0 using LR Clonase II (Thermo Fisher Scientific). The primers used in cloning are listed in Supplementary Table 1.

Transgenic lines were generated by floral dipping method through Agrobacterium tumefaciens infection (GV3101; Clough and Bent, 1998). More than 30 BZR1 overexpression lines were identified. Eight lines were examined to detect expression levels of BZR1. Two representative lines were used for further phenotypic analysis.

\section{Phenotypic and Statistical Analyses}

Half-strength Murashige and Skoog (MS) plates without sucrose were prepared and seeds were surface-sterilized as described previously (Wu et al., 2019a). To prepare ABA-containing plates, 0.3 or $0.4 \mu \mathrm{M}$ ABA were added to half-strength MS medium after sterilization in an autoclave (Sigma-Aldrich; 14375-45-2). For ABA- and BR-containing 1/2 MS plates, the $0.4 \mu \mathrm{M} \mathrm{ABA-}$ containing 1/2 MS medium was supplemented with 10 or $20 \mathrm{nM}$ 
Brassinolide (Furashino; lot no.A122101). Solvent alone was used as a negative control. Bleach-sterilized seeds were sown on plates using a pipette under a clean bench. These plates were placed at $4^{\circ} \mathrm{C}$ for 3 days to stratify seeds before being transferred to a growth chamber at $22^{\circ} \mathrm{C}$ under continuous light. The seed germination ratios were investigated after 12, 24, $36,48,60$, and $72 \mathrm{~h}$ of incubation. The seedling establishment ratios of WT and jmj30-2 jmj32-1 were counted on the seventh day. The mean and SE were determined from three technical replicates ( 3 plates containing 36 plants) from three independent experiments. The photos were taken on a DSC-TX30 camera (SONY). Statistically significant differences in seed germination and seedling establishment were tested using a Chi-squared test.

\section{BR Treatment}

For reverse transcription quantitative polymerase chain reaction (RT-qPCR) and GUS staining analyses, 36-h-old seedlings on 1/2 MS plates were immersed in $1 \mu \mathrm{M}$ BR (Wang et al., 2018a). For RT-qPCR, the seedlings were harvested at 0,3 , and $5 \mathrm{~h}$ after treatment. For GUS staining, seedlings were treated for $5 \mathrm{~h}$.

\section{GUS Staining}

GUS staining was performed as previously described with minor modifications (Sun et al., 2009). Tissues were fixed in $90 \%$ cold acetone for $15 \mathrm{~min}$, rinsed with sterilized $\mathrm{ddH}_{2} \mathrm{O}$ and GUS staining solution without X-Gluc, and incubated with GUS staining solution without $2 \mathrm{mM} \mathrm{X-Gluc} \mathrm{[50} \mathrm{mM}$ $\mathrm{NaPO}_{4}, 0.5 \mathrm{mM} \mathrm{K}{ }_{3} \mathrm{Fe}(\mathrm{CN})_{6}, 0.5 \mathrm{mM} \mathrm{K} \mathrm{K}_{4} \mathrm{Fe}(\mathrm{CN})_{6}$ at $\mathrm{pH}$ 7.2]. pJMJ30:JMJ30-GUS and PJMJ32:JMJ32-GUS were stained at $37^{\circ} \mathrm{C}$ for $2 \mathrm{~h}$ and overnight, respectively. The pigments from the GUSstained tissues were removed using 70\% EtOH treatment for 1 week. The tissues were photographed using an Axio Scope A1 microscope (ZEISS).

\section{RT-qPCR}

Reverse transcription quantitative polymerase chain reaction was performed as previously described (Wu et al., 2019a). Briefly, total RNA was isolated using an RNeasy Plant Mini Kit (Qiagen). cDNA was synthesized from $2.5 \mu \mathrm{g}$ DNase-treated RNA, using PrimeScript RT Master Mix (Takara). RT-qPCR was conducted with gene-specific primers and FastStart DNA essential DNA Green Master (Roche), using a Light Cycler 480 (Roche). EIF4A1 (AT3G13920) was used as an internal control. Each experiment included four technical replicates and three biological replicates of each sample. RT-qPCR primers are described in Supplementary Table 1.

\section{Transcriptomic Analysis}

The transcriptomic data were sourced from previously published data and public databases (Song et al., 2016; Yang et al., 2016; Wu et al., 2019a). Differentially expressed genes (DEGs) were defined by a $p<0.05$. The Venn diagrams were made using jevenn online software. ${ }^{1}$ Heatmaps were made using mev software. ${ }^{2}$ Go analyses were performed using agriGOv2.0 (Tian et al., 2017).

${ }^{1}$ http://jvenn.toulouse.inra.fr/app/example.html

${ }^{2}$ http://mev.tm4.org/

\section{Chromatin Immunoprecipitation}

Chromatin immunoprecipitation (ChIP) was performed as previously described with minor modifications (Yamaguchi et al., 2014). Briefly, $600 \mathrm{mg}$ of 36-h-old seedlings were used for total chromatin extraction. The extracted chromatin was immunoprecipitated using $5 \mu \mathrm{L} /$ sample of anti-HA antibody (Roche; 12CA5) or $5 \mu \mathrm{L} /$ sample of anti-H3K27me3 antibody (Abcam; ab6002). After reverse-crosslinking under $65^{\circ} \mathrm{C}$ overnight and DNA purification, the amount of DNA was quantified by qPCR. ACT7 (AT5G09810) was used as an internal control. For both H3K27me3- and HA-ChIP, all samples were normalized to the input DNA, and the mock-treated wild type was set to 1.0 to obtain the fold change for the other samples. Three independent experiments for each sample were conducted for ChIP-qPCR analysis and four technical replicates were used in each experiment. Statistically significant differences were calculated using a one-way ANOVA followed by post hoc Tukey's HSD test. ChIP-qPCR primers are described in Supplementary Table 1 .

\section{RESULTS}

\section{JMJ30 Expression Is Repressed by BR During the Post-germination Stage}

JMJ30 expression is induced by ABA during post-germination stage (Wu et al., 2019a). To investigate the effect of BR on JMJ30 expression during this stage, 36-h-old wild-type plants were treated with exogenous BR. JMJ30 was under developmental control; its expression levels increased with plant growth (mock $0 \mathrm{~h}$ vs. mock $3 \mathrm{~h}: p=1.2 \times 10^{-6}$; mock $3 \mathrm{~h}$ vs. mock $5 \mathrm{~h}$ : $p=7.0 \times 10^{-3}$ by two-tailed Student's $t$-test (Figure 1A; Wu et al., 2019a). This upregulation was significantly suppressed in BR-treated seedlings within $3 \mathrm{~h}$ after treatment, compared to that in mock-treated seedlings (mock $3 \mathrm{~h}$ vs. BR $3 \mathrm{~h}: p=1.6 \times 10^{-5}$ by two-tailed Student's $t$-test; Figure 1A). The JMJ30 expression levels also remained low at $5 \mathrm{~h}$ after the BR treatment (mock $5 \mathrm{~h}$ vs. BR $5 \mathrm{~h}: p=2.5 \times 10^{-4}$ by two-tailed Student's t-test; Figure 1A). JMJ32 expression was not affected by the presence of BR ( $p>0.05$; Figure 1B). The BR receptor-encoding BRI1 gene was used as a positive control for BR treatment (Supplementary Figure 1).

To confirm that BR suppressed JMJ30 during postgermination stage, we examined spatial patterns of JMJ30-GUS accumulation under BR treatment in a pJMJ30:JMJ30-GUS line (Wu et al., 2019a). In the absence of BR, JMJ30-GUS was expressed mainly in the root meristematic and maturation zones (Figure 1C). Upon BR treatment, JMJ30-GUS levels decreased when compared with the mock-treated control (Figure 1C). We also examined JMJ32-GUS levels with and without BR treatment. Under both conditions, JMJ32-GUS accumulated in cotyledons and root tips at similar levels (Gan et al., 2014). In agreement with the RT-qPCR result, JMJ32 levels remained unchanged by BR treatment (Figure 1D). Thus, while JMJ32 maintains basal levels of stable expression, JMJ30 expression is suppressed by BR during post-germination stage. 


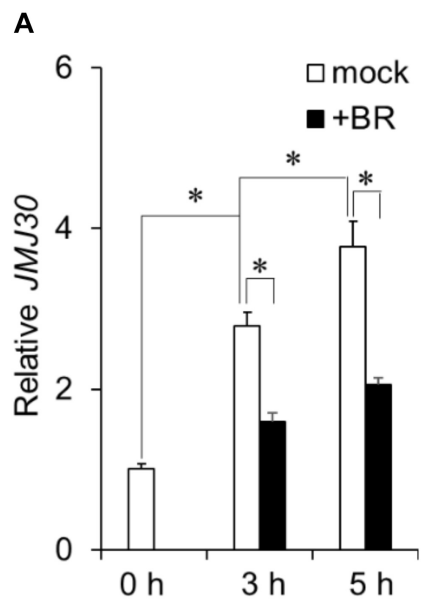

B

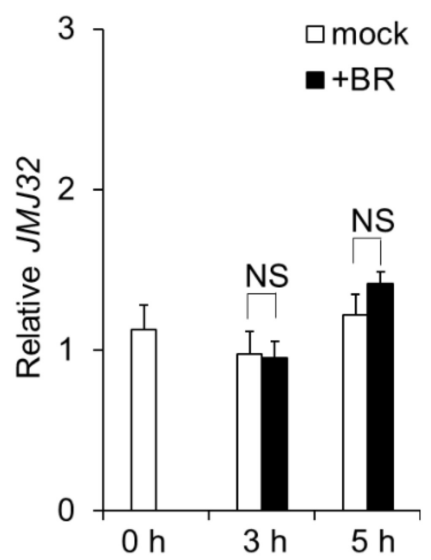

C
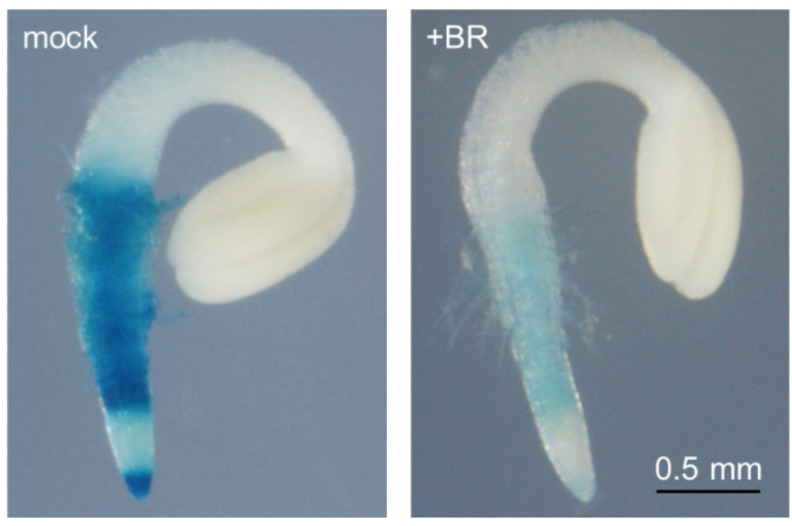

D
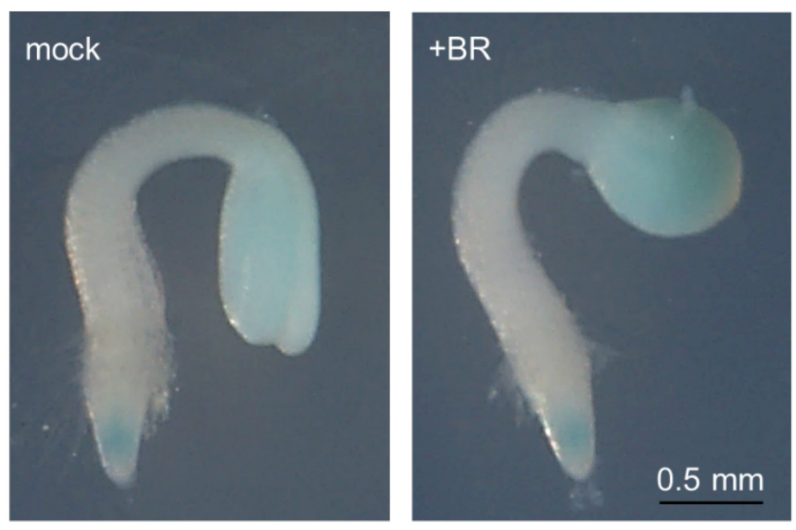

FIGURE 1 | Exogenous BR application suppresses JMJ30 upregulation during post-germination stage. (A,B) Expression of JMJ30 (A) and JMJ32 (B) based on RT-qPCR in wild-type seedlings in response to BR during post-germination stage. Data are from three independent experiments. Values represent means \pm SEM. Asterisks indicate significant differences between two samples based on a two-tailed Student's t-test. (C,D) pJMJ30:JMJ30-GUS (C) and pJMJ32:JMJ32-GUS (D) expression in wild-type background $5 \mathrm{~h}$ after mock or BR treatment. Scale bar $=0.5 \mathrm{~mm}$.

To test the role of JMJ on the crosstalk between ABA and $\mathrm{BR}$, we examined the expression of ABA-inducible genes such as $A B I 5, A B F 2, A B F 3$, and $A B F 4$ (Finkelstein and Lynch, 2000; Kang et al., 2002; Kim et al., 2004; Yoshida et al., 2010) after BR treatment. In wild-type, jmj30-2, and jmj32-1 seedlings, BR inhibited the expression of all four genes; in jmj30-2 jmj321 double mutants, BR repressed these genes to a lesser extent $(p<0.05$ by one-way ANOVA test; jmj30-1 jmj32-1 $+5 \mathrm{~h}$ BR vs. the other samples: $p<0.05$ by post hoc Tukey's HSD test; Supplementary Figure 2). Hence, JMJ30 and JMJ32 may redundantly act as important regulators that link the BR and ABA pathways.

\section{JMJs Are Required for Proper BR Signaling to Inhibit ABA Pathway}

Although jmj30 and jmj32 single mutants showed normal ABA responses, the jmj30 jmj32 double mutants were insensitive to ABA (Wu et al., 2019a). To understand the role of JMJ30 and
JMJ32 on the ABA-BR crosstalk during post-germination stage, we characterized the phenotypic differences between wild type, jmj30-2, jmj32-1, and jmj30-2 jmj32-1 double mutants after exogenous ABA- and BR-treatments.

In the absence of ABA, almost all seedlings developed normally to form green cotyledons, a pair of true leaves, and copious root hairs not only in the wild type, but also in jmj302 jmj32-1 double mutants (Figure 2A). In the presence of $0.3 \mu \mathrm{M}$ ABA, approximately $80 \%$ of germinated wild-type plants failed to develop green cotyledons and the first pair of true leaves, and were arrested just after germination (Figures 2A,B). As reported previously, the jmj30 jmj32 double mutant showed insensitive to $0.3 \mu \mathrm{M}$ ABA treatment as compared with the wild type (ABA-treated WT and ABA-treated jmj30 jmj32: $p<0.05$ by one-way ANOVA test; ABA-treated WT vs. the other samples: $p<0.05$ by post hoc Tukey's HSD test; Figures 2A,B; Wu et al., 2019a). ABA inhibits seedling establishment in the wild type, which is partially restored by BR (Figures 2A,B; Ryu et al., 2014). This restoration suggests that BR suppresses 
ABA signaling during the post-germination stage. Although there is no phenotype in either of the jmj30 and jmj32 single mutants (Supplementary Figure 3), we observed that ABAtreated double mutants and ABA/BR-treated wild type had similar seedling establishment rates, suggesting that mutations in the JMJ genes and exogenous BR treatment have the similar effects in terms of inhibiting ABA signaling (ABA/BR-treated WT and ABA/BR-treated jmj30 jmj32: $p<0.05$ by one-way ANOVA test; Figures 2A,B). We confirmed these results by measuring the fresh weight of ABA/BR-treated wild type and ABA-treated double mutants and monitoring the expression of the ABA-regulated gene $A B I 5$ (Figures 2C,D). Thus, we conclude that JMJ30 and JMJ32 are redundantly required for proper BR signaling to inhibit ABA pathway.

We also examined the effects of JMJ30 and JMJ32 on ABA responses during seed germination. The timing of germination was similar in wild-type, jmj30-2, jmj32-1, and jmj30-2 jmj32-1 seedlings following $\mathrm{ABA}$ and $\mathrm{BR}$ treatment, suggesting that $\mathrm{ABA}$ and BR treatment do not mediate the growth-arrest phenotype (Supplementary Figure 4).

\section{JMJ30 Removes H3K27me3 From the BZR1 Loci in Response to ABA}

Because JMJ mediates the crosstalk between ABA and BR, we hypothesized that JMJ30 and JMJ32 might act on BRrelated downstream targets. To test this hypothesis, we reanalyzed a list of DEGs between mock- and ABA-treated jmj302 jmj32-1 double mutant. Previous RNA sequencing (RNASeq) identified $60 \mathrm{DEGs}$ in jmj30 jmj32 with ABA treatment (Wu et al., 2019a). The top seven Gene Ontology (GO) terms contain two hormone-related descriptions: "Response to hormone stimulus" and "Cellular response to hormone stimulus". The BZR1 gene, a master transcription factor of $\mathrm{BR}$ signaling was found in six out of these seven GO term categories (Figures 3A,B and Supplementary Table 2; Ryu et al., 2007). RT-qPCR analysis confirmed that BZR1 expression was upregulated after ABA treatment in wild type, but not in mock- and ABA-treated jmj30 jmj32 mutants (Figure 3C). Thus, we focused on BZR1 regulation by JMJ30 and JMJ32.

To test if JMJ30 and JMJ32 upregulate BZR1 in response to $\mathrm{ABA}$ through histone demethylation, we first measured the $\mathrm{H} 3 \mathrm{~K} 27 \mathrm{me} 3$ levels in the BZR1 locus. In a published genomewide ChIP-seq dataset, the gene body of the BZR1 was covered with $\mathrm{H} 3 \mathrm{~K} 27 \mathrm{me} 3$ repressive marks in the absence of hormones, in both wild type and jmj30 jmj32 ref6 elf6 mutants (Figure 3D; Yamaguchi et al., 2020). H3 levels were also similar in these samples (Figure 3D). To confirm the effects of ABA treatment on $\mathrm{H} 3 \mathrm{~K} 27 \mathrm{me} 3$ levels, we performed a ChIP assay followed by quantitative PCR (ChIP-qPCR). We detected H3K27me3 marks in the gene body at the BZR1 locus (fragments P3 and P4) in mock-treated wild type and jmj30-2 jmj32-1 mutants (Figure 3E). Upon ABA treatment, these marks decreased in the wild type, but not in jmj30-2 jmj32-1 mutants (P3 and P4: $p<0.05$ by oneway ANOVA test; ABA-treated wild type vs. the other samples: $p<0.05$ by post hoc Tukey's HSD; Figure 3F).
To further test if JMJ30 directly regulates BZR1, we carried out ChIP-qPCR with a biologically active JMJ30 protein tagged with the human influenza hemagglutinin (HA) epitope-the pJMJ30:JMJ30-HA line. Consistent with the H3K27me3 ChIP result, we detected JMJ30-HA association within the promoter and gene body of BZR1 only in the presence of ABA (P2, P3, and P4: $p<0.05$ by one-way ANOVA test; ABA-treated JMJ30HA vs. the other samples: $p<0.05$ by post hoc Tukey's HSD test; Figure 3G). These results indicate that JMJ30 removes $\mathrm{H} 3 \mathrm{~K} 27 \mathrm{me} 3$ at the BZR1 locus in the presence of ABA.

\section{Reduced JMJ Activity and BZR1 Overexpression Synergistically Promote Plant Growth and Development in the Presence of ABA}

To further understand the relationship between JMJs and BZR1, we examined how ectopic expression of BZR1 could affect the ABA insensitivity of jmj30-2 jmj32-1 double mutants. We overexpressed BZR1 under the control of the Cauliflower mosaic virus $35 S$ promoter $(B Z R 1 o x)$ in the double mutants. Of the eight independent lines, jmj30-2 jmj32-1 BZR1 ox line 1 (L1) had a similar expression level to the ABA-treated wild type (Supplementary Figure 5). We also identified six jmj30-2 jmj321 BZR1 ox lines that possessed $\sim 20$-fold (L2-L7) to over 40-fold (L8) increases in the amount of BZR1 transcripts relative to that of ABA-treated wild-type seedlings (Supplementary Figure 5). Consistent with previous findings (Yang et al., 2016), BZR1 ox L1 and L8 lines displayed strong and weak germination defects, respectively (Supplementary Figure 6). We used the L1 and L8 lines for further phenotypic analyses.

In the absence of ABA, wild type, jmj30-2 jmj32-1, and jmj30-2 jmj32-1 BZR1 ox L1 and L8 plants grew normally after germination (Figure 4A). The double mutants had higher seedling establishment rates after $0.3 \mu \mathrm{M}$ ABA treatment, compared with wild type, as reported previously (Figures 4A,B; $\mathrm{Wu}$ et al., 2019a). We observed further increasing seedling establishment rates in jmj30 jmj32 when we overexpress the BZR1 gene $(p<0.01$ by one-way ANOVA test; 0.3 or $0.4 \mu \mathrm{M}$ ABAtreated jmj30 jmj32 vs. the other samples: $p<0.01$ by post hoc Tukey's HSD; Figure 4B). We obtained similar results when we measured fresh weights ( $p<0.01$ by one-way ANOVA test; 0.3 $\mu \mathrm{M}$ or $0.4 \mu \mathrm{M}$ ABA-treated jmj30 jmj32 vs. the other samples: $p<0.01$ by post hoc Tukey's HSD test; Figure 4C) and ABI5 gene expression (Figure 4D). Thus, we conclude that reduced JMJ activity (and hence reduced ABA/SnRK2.8-mediated stress response) and $B Z R 1$ overexpression strongly promote plant growth and development in the presence of ABA.

\section{JMJs Control Cellular Homeostasis During Post-germination Stage by Increasing BZR1 Expression}

Our results suggest that JMJs and BZR1 modulate the same pathway. We hypothesized that JMJs and BZR1 must have common downstream targets if they control ABA-dependent growth arrest. To identify these genes, we conducted computational analysis using public RNA-seq 

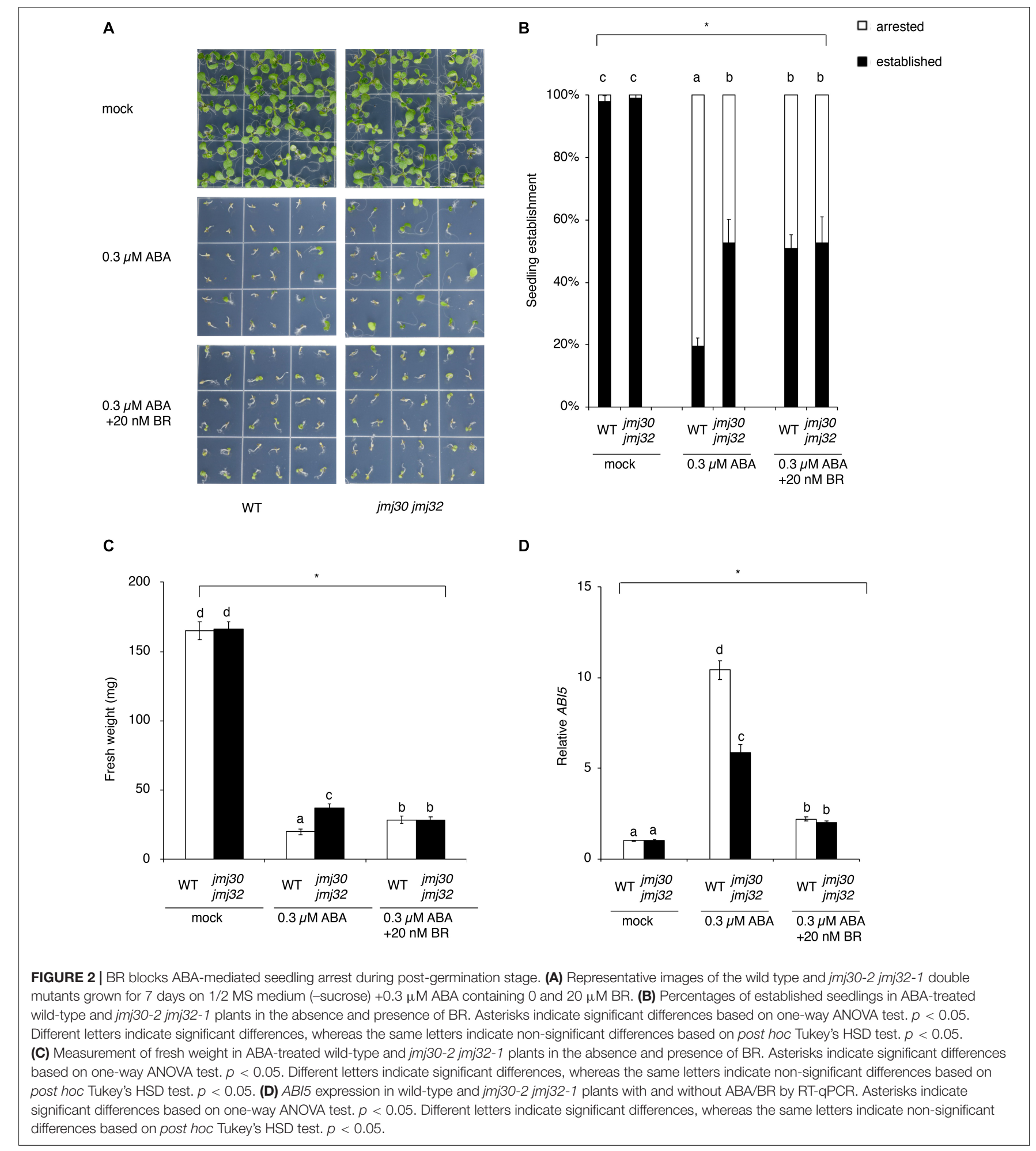

$\square$ arrested

datasets (Yang et al., 2016; Wu et al., 2019a). Although the plant growth conditions of those datasets were not identical, a third transcriptome dataset (Klepikova et al., 2016) supports the conclusion that some genes are expressed at the growth stage investigated in our study (Supplementary Figure 7).
Therefore, we made use of publicly available RNA-seq datasets and identified 20 genes for which expression levels were co-regulated by $J M J s$ and BZR1 (Figures 5A,B). Among these, 12 genes were co-regulated by JMJs and BZR1 and possessed $>1.5$-fold differences relative to controls 
A

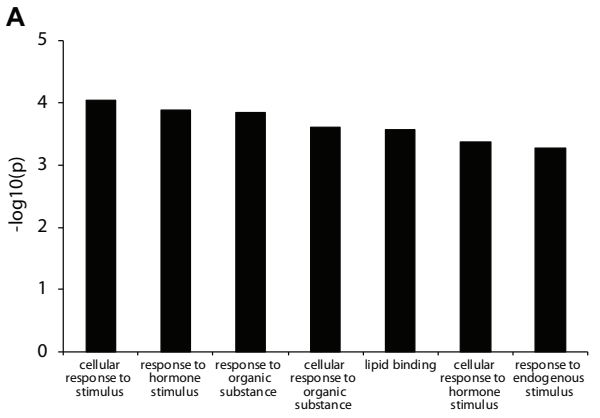

B

\begin{tabular}{ccc}
\hline AGI code & & GO term \\
\hline AT1G75080 & GO:0051716 & cellular response to stimulus \\
BZR1 & GO:0010033 & response to hormone stimulus \\
& GO:0009725 & response to organic substance \\
& GO:0071310 & cellular response to organic substance \\
& GO:0032870 & cellular response to hormone stimulus \\
& GO:0009719 & response to endogenous stimulus \\
response to organic substance & (Wu et al.,2019a) \\
AT1G78290 & GO:0010033 & cellular response to stimulus \\
SnRK2.8 & & response to hormone stimulus \\
AT3G57040 & GO:0051716 & response to organic substance \\
ARR9 & GO:0010033 & GO:0009725 \\
& GO:0071310 & cellular response to organic substance \\
& GO:0032870 & cellular response to hormone stimulus \\
& GO:0009719 & response to endogenous stimulus \\
\hline
\end{tabular}

C

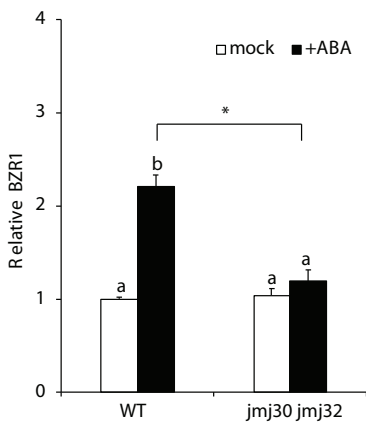

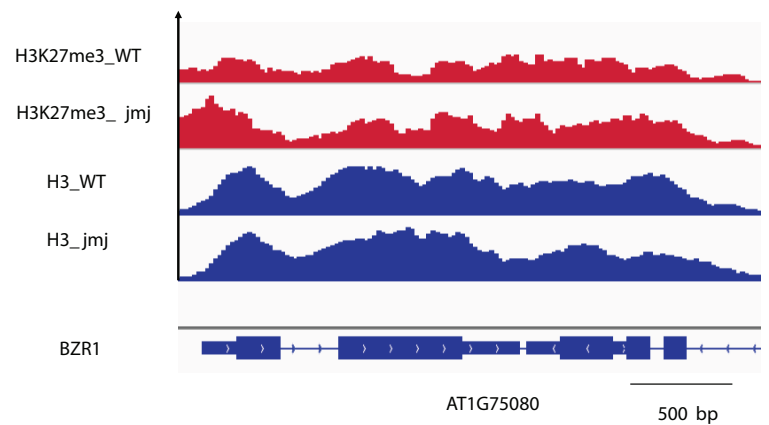

E

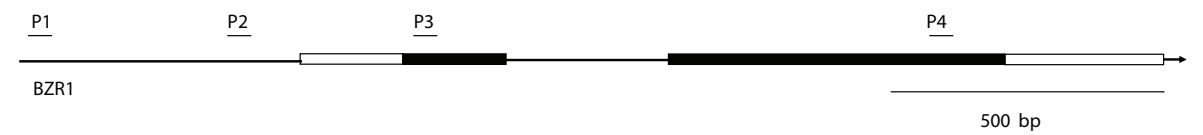

$\mathbf{F}$

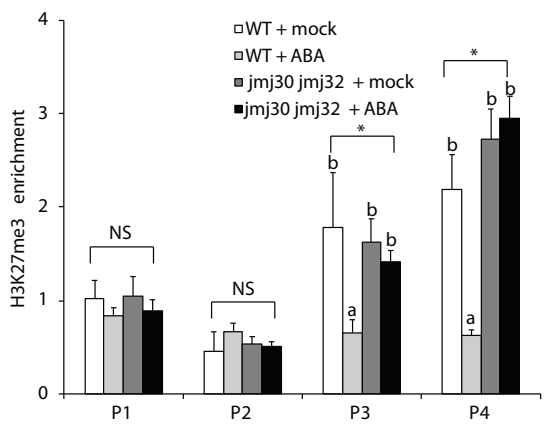

G

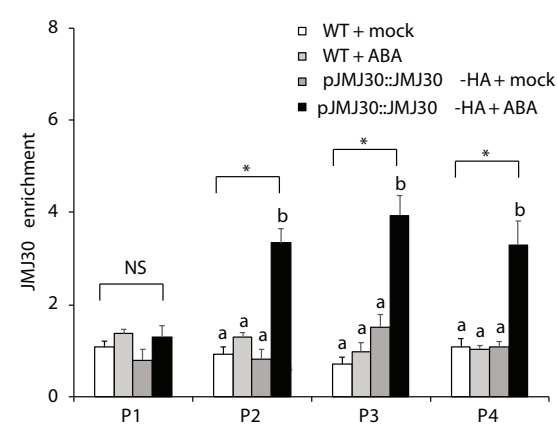

FIGURE 3 | JMJ30 protein removes H3K27me3 from the BZR1 loci in response to ABA. (A) AgriGO analysis revealed seven ABA- and JMJ-dependent biological processes. (B) The GO terms of some plant hormone response genes, such as BZR1, were included. (C) BZR1 expression levels in wild type and jmj30-2 jmj32-1 after ABA treatment, based on RT-qPCR. Asterisks indicate significant differences based on one-way ANOVA test. $p<0.05$. Different letters indicate significant differences, whereas same letters indicate non-significant differences based on post hoc Tukey's HSD test. $p<0.05$. (D) H3K27me3 levels at the BZR1 loci in mock-treated wild type and jmj30-2 jmi32-1. (E) PCR fragments (P) and schematic diagram of genes are shown below the ChIP data. White bars, untranslated regions; black bars, protein coding regions. (F) H3K27me3 ChIP at the BZR1 loci in wild type and jmj30-2 jmj32-1 with and without ABA. (G) JMJ30-HA ChIP at the BZR1 loci in wild type and jmj30-2 jmj32-1 with and without ABA. Values are means \pm SEM from three independent experiments. Asterisks indicate significant differences based on one-way ANOVA test. $p<0.05$. Different letters indicate significant differences, whereas same letters indicate non-significant differences based on a post hoc Tukey's HSD test. $p<0.05$. NS: non-significant.

(Figures 5A,B and Supplementary Table 3). To examine the likely functions of these 12 genes, we tested for GO term enrichment analysis using agriGO. These 12 genes were categorized into "localization," "establishment of localization," and "transport" (Figure 5C).
Figure 5D shows a clustered heatmap of the expression changes observed in two datasets (Song et al., 2016; Wu et al., 2019a). Eight genes were upregulated and four genes were downregulated in wild type following ABA treatment (Figure 5D and Supplementary Table 4). Most of the eight 


\section{A}
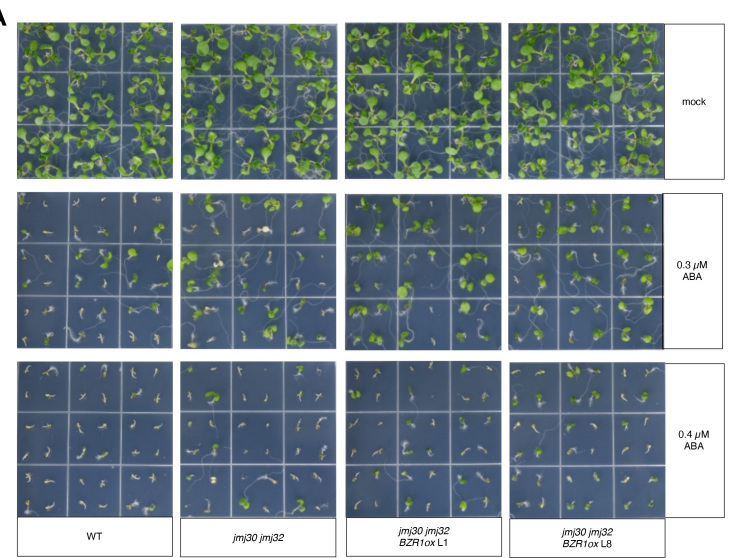

wT

B

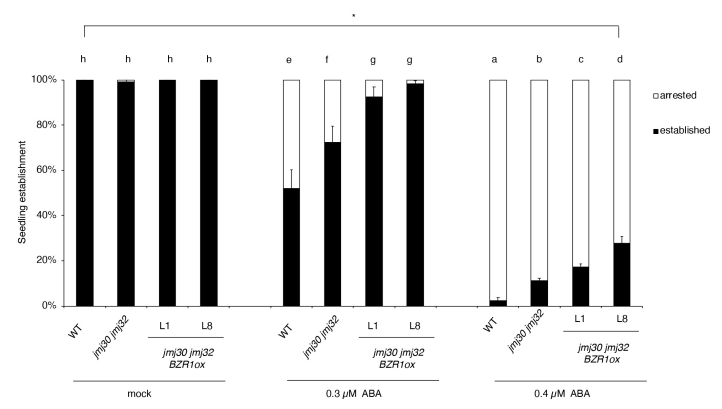

C

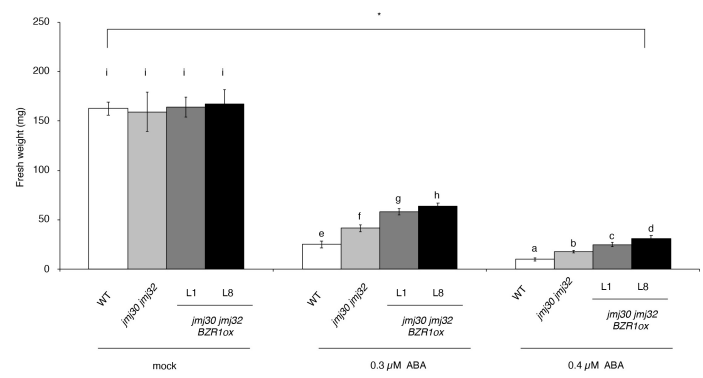

D

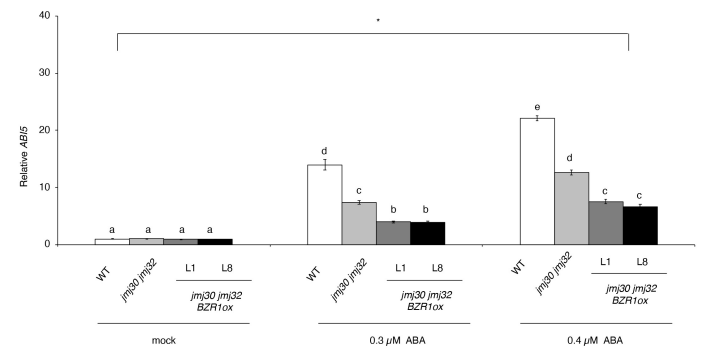

FIGURE 4 | Overexpression of BZR1 in jmj30 jmj32 attenuates ABA sensitivity. (A) Representative images of wild-type, jmj30-2 jmj32-1, jmj30-2 jmj32-1 BZR1ox L1, and jmj30-2 jmj32-1 BZR10x L8 seedlings grown for seven days on $1 / 2$ MS medium (-sucrose) with $0,0.3$, or $0.4 \mu \mathrm{M}$ ABA. (B) Percentage of established and arrested seedlings in wild-type, jmj30-2 jmj32-1, jmj30-2 jmj32-1 BZR1ox L1, and jmj30-2 jmj32-1 BZR1ox L8 plants in the absence and presence of ABA. Asterisks indicate significant differences based on one-way ANOVA test. $p<0.05$. Different letters indicate significant differences based on a post hoc Tukey's HSD test. $p<0.01$. NS: non-significant. (C) Measurement of the fresh weight of wild-type, jmj30-2 jmj32-1, jmj30-2 jmj32-1 BZR1ox L1, and jmj30-2 jmj32-1 BZR1ox L8 seedlings in the absence and presence of ABA. Asterisks indicate significant differences

(Continued)
FIGURE 4 | Continued

based on a one-way ANOVA test. $p<0.05$. Different letters indicate significant differences based on a post hoc Tukey's HSD test. $p<0.01$. NS: non-significant. Values in panels $\mathbf{( B , C )}$ represent means \pm SEM of 36 plants. (D) ABI5 expression in wild-type, jmj30-2 jmi32-1, jmj30-2 jmj32-1 BZR1ox L1, and jmj30-2 jmj32-1 BZR1ox L8 seedlings with and without ABA/BR by RT-qPCR. Asterisks indicate significant differences based on one-way ANOVA test. $p<0.05$. Different letters indicate significant differences, whereas the same letters indicate non-significant differences based on post hoc Tukey's HSD test. $p<0.05$.

genes co-regulated by JMJs and $\mathrm{ABA}$ had reached their highest expression levels at $4 \mathrm{~h}$ after $\mathrm{ABA}$ treatment. These eight genes contain three transport proteins: a regulator of vacuole morphology, REGULATOR OF BULB BIOGENESIS1 (RBB1); and two lipid-transfer proteins, PLASMA-MEMBRANE ASSOCIATED CATION-BINDING PROTEIN1 (PCAP1) and $P C A P 2$ Each of these plays a unique role in transport (Figure 5D). A cytokinin responsive regulator, SULFOTRANSFERASE 4C (ST4C) was identified.

To confirm the RNA-seq results, we examined the expression of these downstream genes in the presence and absence of ABA during post-germination stage. We chose the above four genes because RBB1, PCAP1, and PCAP2 are linked to transport- and establishment of localization-related GO terms, and ST4C is involved in cytokinin response (Lee et al., 2007; Kato et al., 2010; Han et al., 2015; Nagata et al., 2016). Without ABA treatments, there were no expression differences in these genes between wild type and double mutants (Figures $5 \mathrm{E}-\mathbf{H}$ ). In the presence of $\mathrm{ABA}$, the expression of all four genes was significantly higher in the wild type than in the double mutants $(p<0.05$ by oneway ANOVA test; WT + ABA vs. the other samples: $p<0.05$ by post hoc Tukey's HSD; Figures 5E-H). Thus, JMJs and BZR1 may co-regulate genes that are involved in cellular homeostasis during the post-germination stage.

\section{BZR1 Also Regulates ABA/BR Response in a JMJ-Independent Pathway}

Because strong BZR1ox together with the mutations in the $J M J$ genes almost fully inhibited ABA signaling during postgermination stage, we hypothesized that BZR1 may function independently of JMJ-mediated histone modification. To test this hypothesis, we first screened JMJ-independent BZR1 downstream genes by comparing DEGs between ABA-treated WT vs ABA-treated jmj30 jmj32 with two transcriptome datasets of BZR1 targets and ABA-/BR co-regulated genes (Wang et al., 2018a). We identified 3316 genes (Figures 6A,B), of which 52 genes were affected by exogenous $\mathrm{ABA} / \mathrm{BR}$ treatment without JMJs function. Because $b z r 1-D$, which is a gain-of-function mutant of BZR1, exhibits constitutive BR responses, gene regulation in bzr1-D and ABA-treated plants are predicted to work in opposite directions. Indeed, we observed this opposite expression pattern for 18 out of 52 genes (Figures 6A,B and Supplementary Table 5). These were involved in "response to stimuli" and "response to abiotic stimuli" based on GO term analysis (Figure 6C). 


\section{A DEGs between ABA-treated DEGs between bin2-1 WT vs ABA-treated jmj30 jmi32 and bzr1-D bin2- (84 genes)

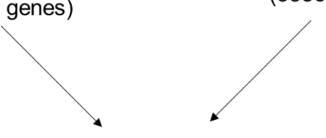 \\ Overlap genes (20 genes)$$
\begin{aligned}
& \text { Co-regulated by JMJs and BZR } \\
& \text { (12 genes ) }
\end{aligned}
$$

C
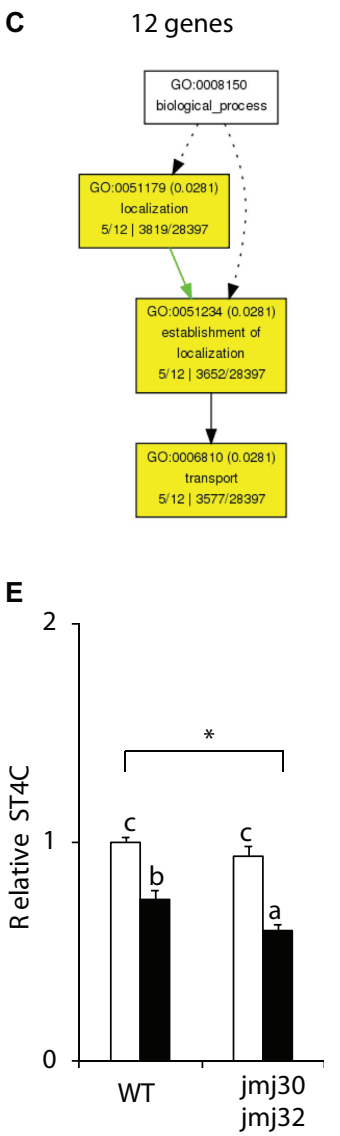

B $\begin{array}{cc}\text { DEGs between ABA } & \text {-treated } \\ \text { WT vs ABA -treated } & \text { DEGs between bin2-1 } \\ \text { jmj30 jmj32 } & \text { and bzr1-D bin2-1 }\end{array}$

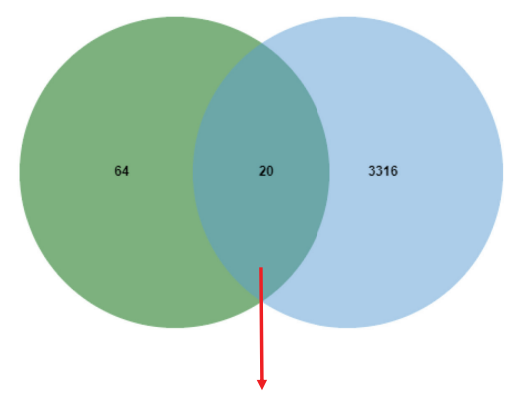

12 genes

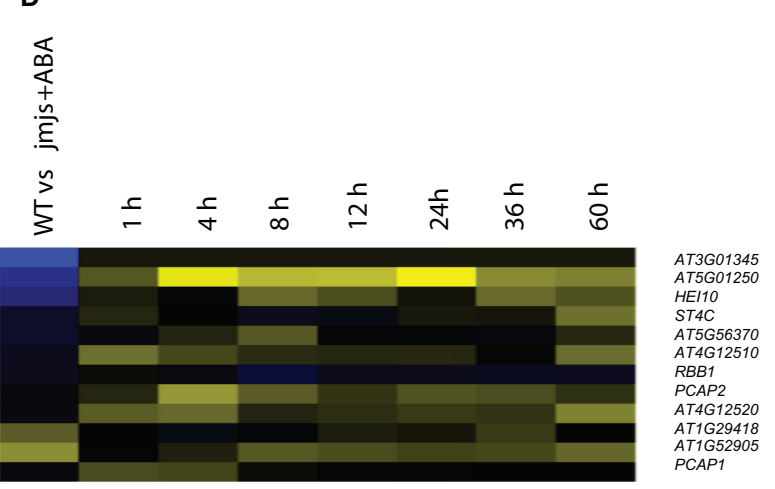

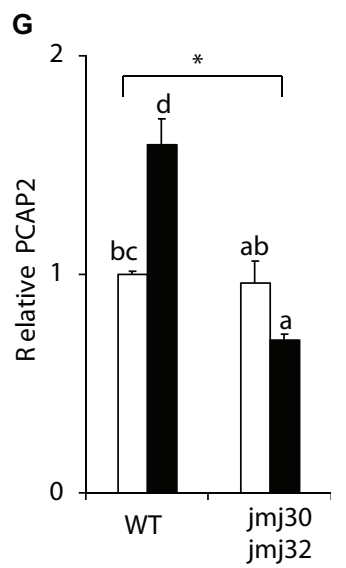

FIGURE 5 | JMJs and BZR1 coordinately regulate transport-related genes. (A) The flowchart of candidate genes selection. (B) Venn diagram showing the number of genes differentially expressed in ABA-treated wild type (WT) and jmj30-2 jmj32-1, and the number of genes differentially expressed in bin2-1 and bzr1-D bin2-1 plants. (C) GO analysis among 12 co-regulated genes. (D) Heatmap displaying the $\log _{2} \mathrm{FC}$ (fold change) of the 12 candidates based on two transcriptome datasets. (E-H) Representative genes in wild-type and jmj30-2 jmj32-1 plants with and without ABA by RT-qPCR: (E) ST4C, (F) PCAP1, (G) PCAP2, and (H) RBB1. Asterisks indicate significant differences based on a one-way ANOVA test. $p<0.05$. Different letters indicate significant differences, whereas same letters indicate non-significant differences based on a post hoc Tukey's HSD test. $p<0.05$.

Of the 18 genes, expression levels of six were temporally regulated during post-germination stage (Song et al., 2016). Among those six genes, two genes-PYRUVATE DECARBOXYLASE 1 (PDC1) and KNOTTED1-LIKE HOMEOBOX GENE 4 (KNAT4)-reached their highest expression levels eight hours after ABA treatment, the expression of AT4G24110 and NRT1/PTR FAMILY 2.13 (NPE2.13) reached their highest expression levels 60 hours after ABA treatment. The PHYTOGLOBIN 1 (AHB1) and WIP DOMAIN PROTEIN 4 (WIP4) expression were inhibit by ABA (Figure 6D). Three of these genes-PDC1, AT4G24110, and AHB1-are linked to "cellular response to hypoxia" (Supplementary Table 6). 


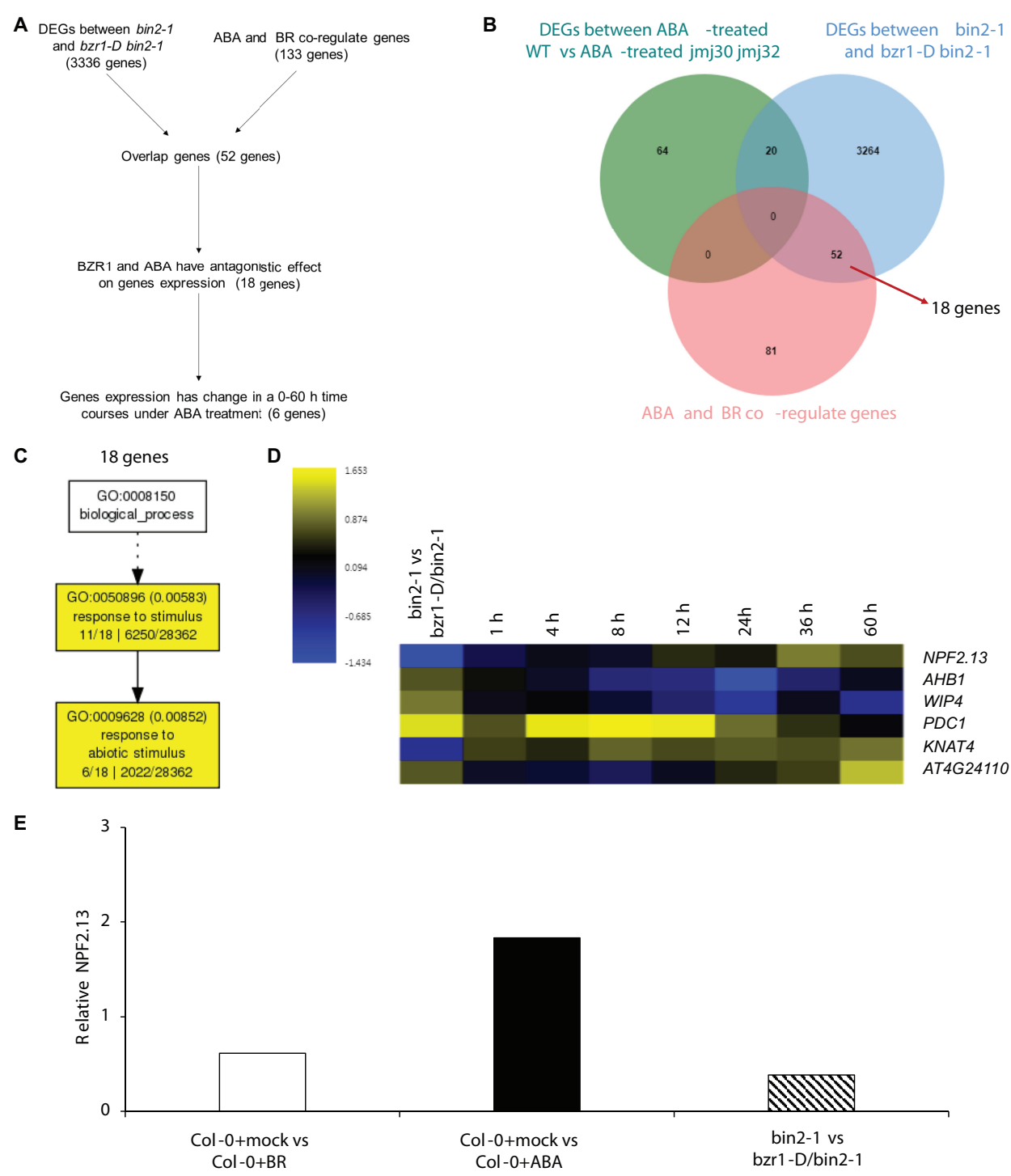

FIGURE 6 | BZR1 regulate ABA/BR responsive genes independently. (A) The flowchart of candidate genes selection. (B) Venn diagram showing the number of genes differentially expressed in ABA-treated wild-type (WT) and jmj30-2 jmj32-1 plants, the number of genes differentially expressed in bin2-1 and bzr1-D bin2-1plants and the number of ABA/BR co-regulated genes. (C) The GO analysis among 18 BZR1 and ABA/BR co-regulated genes. (D) Heatmap displaying the $\log _{2} \mathrm{FC}$ (fold change) of the 18 candidates based on two transcriptome datasets. (E) Relative expression of NPF2.13 by RNA-seq. Y-axis represents FC. $p<0.05$.

NPE2.13 was regulated in the same direction as bzr1-D in BR-treated plants and in the opposite direction in ABA-treated plants (Figure 6E). These results suggest that BZR1 also regulates $\mathrm{ABA} / \mathrm{BR}$-responses during the post-germination stage via a JMJ-independent pathway.

\section{DISCUSSION}

\section{JMJs Play an Important Role in Integrating BR and ABA Crosstalk}

In this study, we found that histone demethylases JMJ30 and JMJ32 integrate inputs from ABA and BR during post-germination stage (Figure 7). Subsequently, JMJ30 and JMJ32 control the expression two downstream targets, SnRK2.8 and BZR1 depending on the hormonal inputs. Since those two genes have opposite functions during post-germination stage, JMJ30 and JMJ32 could determine the balance between stress response and growth by regulating those targets. Furthermore, this crosstalk is regulated by both JMJ-dependent and JMJ-independent pathways.

Our study provides genetic and biochemical evidence to support the role of JMJs in integrating $\mathrm{ABA}$ - and BRsignaling. ABA upregulates JMJ30 and BR downregulates it (Wu et al., 2019a,b). BR-mediated repression of ABA-inducible genes was absent in jmj30 jmj32 mutants (Supplementary Figure 2). Exogenous BR treatment and the mutations in the 


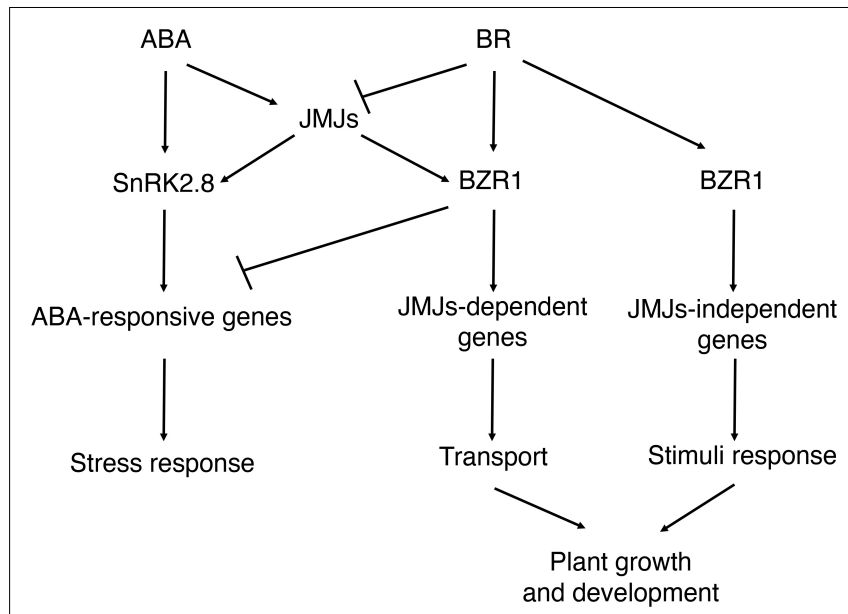

FIGURE 7 | Proposed models for the interaction between ABA and BR during post-germination stage. ABA inhibits the later growth of the seedling. BR promotes the later growth of the seedling. JMJ-dependent and JMJ-independent pathway modulate growth arrest upon perception of ABA and BR signals post-germination.

JMJ30 and JMJ32 genes have the same effects for inhibiting ABA signaling (Figure 2). Although we previously revealed that ABI3 activated JMJ30 expression upon ABA treatment, transcription factors that repress $J M J 30$ expression in response to $\mathrm{BR}$ are not identified, yet. Based on the publicly available ChIP datasets, BZR1 binds to JMJ30. Although the ChIP experiment was conducted in a different plant growth stage, BZR1 could be a regulator of JMJ30. Identification and/or verification of these transcription factors will elucidate how this integration is regulated. Antagonistic relationship on gene expression is often regulated by competitive binding at common regulatory regions. Interestingly, the JMJ30 promoter region around an ABI3 binding site is evolutionally conserved in Brassicaceae spices (Wu et al., 2019a). Thus, such mechanism might also exist for regulating JMJ30 expression by $\mathrm{ABA}$ and $\mathrm{BR}$.

\section{JMJs Directly Regulate Both BR- and ABA-Related Downstream Targets}

We have identified genes critical to ABA-mediated growth arrest, which are regulated through JMJ-dependent histone modification. These genes were linked to many biological processes including hormonal control. $S n R K 2.8$ acts downstream of JMJ30 in ABA-mediated growth arrest (Wu et al., 2019a). In this study, we identified the master regulator of BR signaling, BZR1, as another JMJ30 target (Figure 3 Supplementary Table 2). We have shown that BZR1 is a direct target of JMJs in the presence of ABA (Figure 3). Based on the previous in vitro demethylase assay (Gan et al., 2014), JMJ30 specifically demethylates oligonucleosomes at $\mathrm{H} 3 \mathrm{~K} 27 \mathrm{me} 2 / 3$, but not $\mathrm{H} 3 \mathrm{~K} 9 \mathrm{me} 2 / 3$. Although $\mathrm{H} 3 \mathrm{~K} 9 \mathrm{ac}$ changes were reported in elf6 ref6 mutants by the previous publication (Yu et al., 2008), JMJ30 is likely to demethylate $\mathrm{H} 3 \mathrm{~K} 27 \mathrm{me} 3$. Indeed, $\mathrm{ABA}$-dependent removal of $\mathrm{H} 3 \mathrm{~K} 27 \mathrm{me} 3$ at $B Z R 1$ was regulated via JMJ30 and
JMJ32. Thus, JMJ30 and JMJ32 control the ABA and BR signal transduction pathways by regulating distinct downstream targets to balance growth and stress responses through $\mathrm{H} 3 \mathrm{~K} 27 \mathrm{me} 3$ removal (Figure 7). Overall, H3K27 demethylases tend to have redundant roles during plant development and stress responses (Lu et al., 2011; Gan et al., 2014; Zheng et al., 2019; Yamaguchi et al., 2020). Unlike JMJ30 and JMJ32, ELF6, and REF6 could also control $\mathrm{H} 3 \mathrm{~K} 9 \mathrm{me} 3$ in a direct way by unknown mechanisms (Yu et al., 2008). Further analysis using multiple mutants is required to figure out their functional differences in response to ABA. Our genetic analysis revealed that reduced JMJ30 and JMJ32 activities (and hence reduced ABA-JMJ30-SnRK2.8 pathway) and BZR1 overexpression synergistically promote plant growth and development in the presence of ABA (Figure 4). Furthermore, our results also support that BZR1 represses ABA-responsive gene expression as reported previously (Yang et al., 2016). Not only JMJ, but also BZR1 control ABA-BR crosstalk (Figure 7).

\section{BZR1 Regulates ABA-Mediated Growth Arrest in JMJ-Dependent and JMJ-Independent Pathways}

Phenotypic analysis using BZR1ox and jmj30 jmj32 double mutants suggested the existence of two pathways involved in ABA-dependent growth arrest: JMJdependent and JMJ-independent. We identified genes involved in the two distinct pathways using public transcriptome datasets.

The genes that act in the JMJ-BZR1 pathway are membrane proteins linked to transport (Figure 5 and Supplementary Table 3). The four genes transcriptionally regulated by JMJ and BZR1 include RBB1, PCAP1, PCAP2, and ST4C. The membrane protein RBB1 controls vacuole bulb formation (Han et al., 2015). Bulbs are located in the epidermis of the root and may act as membrane reservoirs for cell expansion (Uemura et al., 2002). PCAP2 is a plasma membrane-associated $\mathrm{Ca}^{2+}$ binding protein (Zhu et al., 2013), which acts in ABA response and in the crosstalk between ABA and salicylic acid (SA) during root growth (Wang et al., 2018b,c). We hypothesize that JMJ and BZR1 co-regulate PCAP2 expression to control the crosstalk between ABA and BR signals. PCAP1, a homolog of $P C A P 2$, is also a plasma membrane-associated $\mathrm{Ca}^{2+}$-binding protein (Tanaka-Takada et al., 2019). Although functional characterization using both homologs has not been conducted yet, PCAP1 may share functions with PCAP2. The cytokinin responsive regulator, $S T 4 C$, is a common target of JMJ and BZR1 (Figure 5E). Cytokinin signaling antagonizes ABA-mediated inhibition of seedling growth (Huang et al., 2018). Overall, these four genes may control root growth in the JMJ-BZR1dependent pathway.

On the other hand, some genes that are linked to stress response function in the BZR1 pathway independently of JMJs (Figure 6 and Supplementary Table 3). These genes are enriched for the GO terms "response to stimuli" and "response to abiotic stimuli" (Figure 6C). Four well-characterized proteins were involved in BZR1 
and JMJ-independent pathways (Figure 6D). Two candidates, $P D C 1$ and $A H B 1$, respond to hypoxic stress. PDC1 enhances plant survival under low oxygen stress (Ismond et al., 2003) and $A H B 1$ reduces $\mathrm{NO}$ emission to help plant escape from hypoxic stress (Perazzolli et al., 2004). Thus, BZR1 may affect gene expression in the hypoxic response pathway to integrate ABA and BR signals. The third candidate, NPF2.13, is responsible for normal nitrogen cycling under low nitrogen stress (Nour-Eldin et al., 2012; Leran et al., 2014). The fourth candidate, KNAT4, was identified as a BZR1 target. Its expression in the elongation zone of the root is triggered by cytokinin and light (Truernit et al., 2006). Therefore, JMJ-independent genes that are modulated by BZR1 may play key roles in various stress responses.

Although we have identified two different pathways, we still don't know their spatial and temporal domains. JMJ30 accumulated mainly in the root meristematic and maturation zones (Figure 1), whereas BZR1 is expressed in the root elongation and maturation zones. Thus, the JMJ-BZR1 pathway might function in the maturation zone of root, where those genes are co-expressed. In contrast, the JMJ-independent BZR1 pathway may function in the root elongation zone. Further investigation is required to specify the exact function of these pathways.

\section{DATA AVAILABILITY STATEMENT}

The original contributions presented in the study are included in the article/Supplementary Material, further inquiries can be directed to the corresponding authors.

\section{AUTHOR CONTRIBUTIONS}

JW, NY, and TI: conceptualization. JW and NY: data curation, formal analysis, investigation, software, visualization, and writing - original draft. MY, NY, and TI: funding acquisition. NY: project administration and validation. NY and TI: supervision. All authors: review and editing.

\section{REFERENCES}

Bechtold, U., and Field, B. (2018). Molecular mechanisms controlling plant growth during abiotic stress. J. Exp. Bot. 69, 2753-2758. doi: 10.1093/jxb/er y157

Blatt, M. R., Brodribb, T. J., and Torii, K. U. (2017). Small pores with a big impact. Plant Physiol. 174, 467-469. doi: 10.1104/pp.17.00642

Cai, Z., Liu, J., Wang, H., Yang, C., Chen, Y., Li, Y., et al. (2014). GSK3-like kinases positively modulate abscisic acid signaling through phosphorylating subgroup III SnRK2s in Arabidopsis. Proc. Natl. Acad. Sci. U.S.A. 111, 9651-9656. doi: 10.1073/pnas.1316717111

Cano-Delgado, A., Yin, Y., Yu, C., Vafeados, D., Mora-Garcia, S., Cheng, J. C., et al. (2004). BRL1 and BRL3 are novel brassinosteroid receptors that function in vascular differentiation in Arabidopsis. Development 131, 5341-5351. doi: 10.1242/dev.01403

\section{FUNDING}

This work was supported by a grant from the National Key Research and Development Program of China (2018YFD1000904) and the Scientific Research Fund of Hunan Provincial Education Department, China (17K035 and 15K046) to MY, and the Japan Science and Technology Agency "PREST" (JPMJPR15QA), a JSPS KAKENHI Grant-in-Aid for Scientific Research on Innovative Areas (No. 18H04782), a JSPS KAKENHI Grant-in-Aid for Scientific Research B (No. 18H02465), a Grantin-Aid for challenging Exploratory Research (No. 19K22431), and a grant from the Mishima Kaiun Memorial Foundation to NY, and a JSPS KAKENHI Grant-in-Aid for Scientific Research on Innovative Areas (Nos. 19H04865 and 20H04888), a JSPS KAKENHI Grant-in-Aid for Scientific Research A (Nos. 15H02405 and 20H00470), and a Grant-in-Aid for challenging Exploratory Research (No. 18K19342) to TI.

\section{ACKNOWLEDGMENTS}

We thank Akie Takahashi, Hiroko Egashira, Kyoko Sunuma, Mayumi Nara, Mikiko Higashiura, Taeko Kawakami, and Yuka Kadoya for technical assistance.

\section{SUPPLEMENTARY MATERIAL}

The Supplementary Material for this article can be found online at: https://www.frontiersin.org/articles/10.3389/fpls.2020. 596835/full\#supplementary-material

Supplementary Table 1 | Primers used in this study.

Supplementary Table 2 | DEGs are from seven GO term.

Supplementary Table 3 | 12DEGs co-regulated by JMJs and BZR1.

Supplementary Table 4 | 12 candidates expression change under ABA-treated time courses.

Supplementary Table 5 | 18 genes expression were regulated by BZR1 and ABA/BR treatment.

Supplementary Table 6 | Six candidates expression change under ABA-treated time courses.

Clough, S. J., and Bent, A. F. (1998). Floral dip: a simplified method for Agrobacterium-mediated transformation of Arabidopsis thaliana. Plant J. 16, 735-743. doi: 10.1046/j.1365-313x.1998.00343.x

Clouse, S. D. (2011). Brassinosteroid signal transduction: from receptor kinase activation to transcriptional networks regulating plant development. Plant Cell 23, 1219-1230. doi: 10.1105/tpc.111.084475

Clouse, S. D., Langford, M., and McMorris, T. C. (1996). A brassinosteroidinsensitive mutant in Arabidopsis thaliana exhibits multiple defects in growth and development. Plant Physiol. 111, 671-678. doi: 10.1104/pp.111.3.671

Cutler, S. R., Rodriguez, P. L., Finkelstein, R. R., and Abrams, S. R. (2010). Abscisic acid: emergence of a core signaling network. Annu. Rev. Plant Biol. 61, 651-679.

Fabregas, N., Li, N., Boeren, S., Nash, T. E., Goshe, M. B., Clouse, S. D., et al. (2013). The brassinosteroid insensitivel-like3 signalosome complex regulates Arabidopsis root development. Plant Cell 25, 3377-3388. doi: 10.1105/tpc.113. 114462 
Finkelstein, R. (2013). Abscisic acid synthesis and response. Arabidopsis Book 11:e0166. doi: 10.1199/tab.0166

Finkelstein, R. R., and Lynch, T. J. (2000). The Arabidopsis abscisic acid response gene ABI5 encodes a basic leucine zipper transcription factor. Plant Cell 12, 599-609. doi: 10.1105/tpc.12.4.599

Foyer, C. H., Kiddle, G., and Verrier, P. (2007). Transcriptional profiling approaches to understanding how plants regulate growth and defence: a case study illustrated by analysis of the role of vitamin C. EXS 97, 55-86. doi: 10.1007/978-3-7643-7439-6_3

Gan, E. S., Xu, Y., Wong, J. Y., Goh, J. G., Sun, B., Wee, W. Y., et al. (2014). Jumonji demethylases moderate precocious flowering at elevated temperature via regulation of FLC in Arabidopsis. Nat. Commun. 5:5098. doi: 10.1038/ ncomms6098

Gruszka, D. (2013). The brassinosteroid signaling pathway-new key players and interconnections with other signaling networks crucial for plant development and stress tolerance. Int. J. Mol. Sci. 14, 8740-8774. doi: 10.3390/ijms 14058740

Han, S. W., Alonso, J. M., and Rojas-Pierce, M. (2015). REGULATOR OF BULB BIOGENESIS1 (RBB1) is involved in vacuole bulb formation in Arabidopsis. PLoS One 10:e0125621. doi: 10.1371/journal.pone.0125621

Hrabak, E. M., Chan, C. W., Gribskov, M., Harper, J. F., Choi, J. H., Halford, N., et al. (2003). The Arabidopsis CDPK-SnRK superfamily of protein kinases. Plant Physiol. 132, 666-680. doi: 10.1104/pp.102.011999

$\mathrm{Hu}, \mathrm{Y}$., and $\mathrm{Yu}, \mathrm{D}$. (2014). BRASSINOSTEROID INSENSITIVE2 interacts with ABSCISIC ACID INSENSITIVE5 to mediate the antagonism of brassinosteroids to abscisic acid during seed germination in Arabidopsis. Plant Cell 26, 4394-4408. doi: 10.1105/tpc.114.130849

Huang, X., Hou, L., Meng, J., You, H., Li, Z., Gong, Z., et al. (2018). The antagonistic action of abscisic acid and cytokinin signaling mediates drought stress response in Arabidopsis. Mol. Plant 11, 970-982. doi: 10.1016/j.molp.2018.05.001

Ibanez, C., Delker, C., Martinez, C., Burstenbinder, K., Janitza, P., Lippmann, R., et al. (2018). Brassinosteroids dominate hormonal regulation of plant thermomorphogenesis via BZR1. Curr. Biol. 28, 303-310.e3. doi: 10.1016/j.cub. 2017.11.077

Ismond, K. P., Dolferus, R., de Pauw, M., Dennis, E. S., and Good, A. G. (2003). Enhanced low oxygen survival in Arabidopsis through increased metabolic flux in the fermentative pathway. Plant Physiol. 132, 1292-1302. doi: 10.1104/pp. 103.022244

Kang, J. Y., Choi, H. I., Im, M. Y., and Kim, S. Y. (2002). Arabidopsis basic leucine zipper proteins that mediate stress-responsive abscisic acid signaling. Plant Cell 14, 343-357. doi: $10.1105 /$ tpc.010362

Kato, M., Nagasaki-Takeuchi, N., Ide, Y., and Maeshima, M. (2010). An Arabidopsis hydrophilic Ca2(+) -binding protein with a PEVK-rich domain, $\mathrm{PCaP} 2$, is associated with the plasma membrane and interacts with calmodulin and phosphatidylinositol phosphates. Plant Cell Physiol. 51, 366-379. doi: 10. 1093/pcp/pcq003

Kim, J. M., To, T. K., Ishida, J., Morosawa, T., Kawashima, M., Matsui, A., et al. (2008). Alterations of lysine modifications on the histone $\mathrm{H} 3 \mathrm{~N}$-tail under drought stress conditions in Arabidopsis thaliana. Plant Cell Physiol. 49, 1580-1588. doi: $10.1093 / \mathrm{pcp} / \mathrm{pcn} 133$

Kim, S., Kang, J. Y., Cho, D. I., Park, J. H., and Kim, S. Y. (2004). ABF2, an ABRE-binding bZIP factor, is an essential component of glucose signaling and its overexpression affects multiple stress tolerance. Plant J. 40, 75-87. doi: $10.1111 /$ j.1365-313X.2004.02192.x

Klepikova, A. V., Kasianov, A. S., Gerasimov, E. S., Logacheva, M. D., and Penin, A. A. (2016). A high resolution map of the Arabidopsis thaliana developmental transcriptome based on RNA-seq profiling. Plant J. 88, 1058-1070. doi: 10.1111/ tpj. 13312

Lafos, M., Kroll, P., Hohenstatt, M. L., Thorpe, F. L., Clarenz, O., and Schubert, D. (2011). Dynamic regulation of H3K27 trimethylation during Arabidopsis differentiation. PLoS Genet. 7:e1002040. doi: 10.1371/journal.pgen.1002040

Lee, D. J., Park, J. Y., Ku, S. J., Ha, Y. M., Kim, S., Kim, M. D., et al. (2007). Genome-wide expression profiling of ARABIDOPSIS RESPONSE REGULATOR 7(ARR7) overexpression in cytokinin response. Mol. Genet. Genomics 277, 115-137. doi: 10.1007/s00438-006-0177-x

Leran, S., Varala, K., Boyer, J. C., Chiurazzi, M., Crawford, N., Daniel-Vedele, F., et al. (2014). A unified nomenclature of NITRATE TRANSPORTER 1/PEPTIDE TRANSPORTER family members in plants. Trends Plant Sci. 19, 5-9. doi: 10.1016/j.tplants.2013.08.008
Li, J., and Chory, J. (1997). A putative leucine-rich repeat receptor kinase involved in brassinosteroid signal transduction. Cell 90, 929-938.

Liu, N., Fromm, M., and Avramova, Z. (2014). H3K27me3 and H3K4me3 chromatin environment at super-induced dehydration stress memory genes of Arabidopsis thaliana. Mol. Plant 7, 502-513. doi: 10.1093/mp/ssu001

Lopez-Molina, L., Mongrand, S., McLachlin, D. T., Chait, B. T., and Chua, N. H. (2002). ABI5 acts downstream of ABI3 to execute an ABA-dependent growth arrest during germination. Plant J. 32, 317-328.

Lu, F., Cui, X., Zhang, S., Jenuwein, T., and Cao, X. (2011). Arabidopsis REF6 is a histone H3 lysine 27 demethylase. Nat. Genet. 43, 715-719. doi: 10.1038/ng.854

Luo, M., Liu, X., Singh, P., Cui, Y., Zimmerli, L., and Wu, K. (2012). Chromatin modifications and remodeling in plant abiotic stress responses. Biochim. Biophys. Acta 1819, 129-136. doi: 10.1016/j.bbagrm.2011.06.008

Nagata, C., Miwa, C., Tanaka, N., Kato, M., Suito, M., Tsuchihira, A., et al. (2016). A novel-type phosphatidylinositol phosphate-interactive, Ca-binding protein PCaP1 in Arabidopsis thaliana: stable association with plasma membrane and partial involvement in stomata closure. J. Plant Res. 129, 539-550.

Nakabayashi, K., Okamoto, M., Koshiba, T., Kamiya, Y., and Nambara, E. (2005). Genome-wide profiling of stored mRNA in Arabidopsis thaliana seed germination: epigenetic and genetic regulation of transcription in seed. Plant $J$. 41, 697-709. doi: 10.1111/j.1365-313X.2005.02337.x

Nakashima, K., Fujita, Y., Kanamori, N., Katagiri, T., Umezawa, T., Kidokoro, S., et al. (2009). Three Arabidopsis SnRK2 protein kinases, SRK2D/SnRK2.2, SRK2E/SnRK2.6/OST1 and SRK2I/SnRK2.3, involved in ABA signaling are essential for the control of seed development and dormancy. Plant Cell Physiol. 50, 1345-1363. doi: 10.1093/pcp/pcp083

Nam, K. H., and Li, J. (2002). BRI1/BAK1, a receptor kinase pair mediating brassinosteroid signaling. Cell 110, 203-212.

Nour-Eldin, H. H., Andersen, T. G., Burow, M., Madsen, S. R., Jorgensen, M. E., Olsen, C. E., et al. (2012). NRT/PTR transporters are essential for translocation of glucosinolate defence compounds to seeds. Nature 488, 531-534. doi: 10. 1038/nature11285

Park, S. Y., Fung, P., Nishimura, N., Jensen, D. R., Fujii, H., Zhao, Y., et al. (2009). Abscisic acid inhibits type $2 \mathrm{C}$ protein phosphatases via the PYR/PYL family of START proteins. Science 324, 1068-1071. doi: 10.1126/science.1173041

Perazzolli, M., Dominici, P., Romero-Puertas, M. C., Zago, E., Zeier, J., Sonoda, M., et al. (2004). Arabidopsis nonsymbiotic hemoglobin AHb1 modulates nitric oxide bioactivity. Plant Cell 16, 2785-2794. doi: 10.1105/tpc.104.025379

Qian, S., Lv, X., Scheid, R. N., Lu, L., Yang, Z., Chen, W., et al. (2018). Dual recognition of H3K4me3 and H3K27me3 by a plant histone reader SHL. Nat. Commun. 9:2425. doi: 10.1038/s41467-018-04836-y

Ryu, H., Cho, H., Bae, W., and Hwang, I. (2014). Control of early seedling development by BES1/TPL/HDA19-mediated epigenetic regulation of ABI3. Nat. Commun. 5:4138.

Ryu, H., Kim, K., Cho, H., Park, J., Choe, S., and Hwang, I. (2007). Nucleocytoplasmic shuttling of BZR1 mediated by phosphorylation is essential in Arabidopsis brassinosteroid signaling. Plant Cell 19, 2749-2762. doi: 10.1105/ tpc.107.053728

Sanchez-Bel, P., Sanmartin, N., Pastor, V., Mateu, D., Cerezo, M., Vidal-Albalat, A., et al. (2018). Mycorrhizal tomato plants fine tunes the growth-defence balance upon N depleted root environments. Plant Cell Environ. 41, 406-420. doi: $10.1111 /$ pce. 13105

Shang, Y., Dai, C., Lee, M. M., Kwak, J. M., and Nam, K. H. (2016). BRI1-associated receptor kinase 1 regulates guard cell ABA signaling mediated by open stomata 1 in Arabidopsis. Mol. Plant 9, 447-460. doi: 10.1016/j.molp.2015.12.014

Skubacz, A., Daszkowska-Golec, A., and Szarejko, L. (2016). The role and regulation of ABI5 (ABA-Insensitive 5) in plant development, abiotic stress responses and phytohormone crosstalk. Front. Plant Sci. 7:1884. doi: 10.3389/ fpls.2016.01884

Song, L., Huang, S. C., Wise, A., Castanon, R., Nery, J. R., Chen, H., et al. (2016). A transcription factor hierarchy defines an environmental stress response network. Science 354:aag1550. doi: 10.1126/science.aag1550

Sun, B., Looi, L. S., Guo, S., He, Z., Gan, E. S., Huang, J., et al. (2014). Timing mechanism dependent on cell division is invoked by Polycomb eviction in plant stem cells. Science 343:1248559. doi: 10.1126/science. 1248559

Sun, B., Xu, Y., Ng, K. H., and Ito, T. (2009). A timing mechanism for stem cell maintenance and differentiation in the Arabidopsis floral meristem. Genes Dev. 23, 1791-1804. doi: 10.1101/gad.1800409 
Suzuki, M., Ketterling, M. G., Li, Q. B., and McCarty, D. R. (2003). Viviparous1 alters global gene expression patterns through regulation of abscisic acid signaling. Plant Physiol. 132, 1664-1677.

Tanaka-Takada, N., Kobayashi, A., Takahashi, H., Kamiya, T., Kinoshita, T., and Maeshima, M. (2019). Plasma membrane-associated Ca2+-binding protein $\mathrm{PCaP} 1$ is involved in root hydrotropism of Arabidopsis thaliana. Plant Cell Physiol. 60, 1331-1341. doi: 10.1093/pcp/pcz042

Tian, T., Liu, Y., Yan, H., You, Q., Yi, X., Du, Z., et al. (2017). agriGO v2.0: a GO analysis toolkit for the agricultural community, 2017 update. Nucleic Acids Res. 45, W122-W129. doi: 10.1093/nar/gkx382

Tian, Y., Fan, M., Qin, Z., Lv, H., Wang, M., Zhang, Z., et al. (2018). Hydrogen peroxide positively regulates brassinosteroid signaling through oxidation of the BRASSINAZOLE-RESISTANT1 transcription factor. Nat. Commun. 9:1063. doi: 10.1038/s41467-018-03463-x

Truernit, E., Siemering, K. R., Hodge, S., Grbic, V., and Haseloff, J. (2006). A map of KNAT gene expression in the Arabidopsis root. Plant Mol. Biol. 60, 1-20.

Tsukada, Y., Fang, J., Erdjument-Bromage, H., Warren, M. E., Borchers, C. H., Tempst, P., et al. (2006). Histone demethylation by a family of JmjC domaincontaining proteins. Nature 439, 811-816. doi: 10.1038/nature04433

Uemura, T., Yoshimura, S. H., Takeyasu, K., and Sato, M. H. (2002). Vacuolar membrane dynamics revealed by GFP-AtVam3 fusion protein. Genes Cells 7 , 743-753. doi: 10.1046/j.1365-2443.2002.00550.x

Wang, H., Tang, J., Liu, J., Hu, J., Liu, J., Chen, Y., et al. (2018a). Abscisic acid signaling inhibits brassinosteroid signaling through dampening the dephosphorylation of BIN2 by ABI1 and ABI2. Mol. Plant 11, 315-325. doi: 10.1016/j.molp.2017.12.013

Wang, X., Wang, L., Wang, Y., Liu, H., Hu, D., Zhang, N., et al. (2018b). Arabidopsis PCaP2 plays an important role in chilling tolerance and ABA response by activating $\mathrm{CBF}$ - and $\mathrm{SnRK} 2$-mediated transcriptional regulatory network. Front. Plant Sci. 9:215. doi: 10.3389/fpls.2018.00215

Wang, X., Wang, Y., Wang, L., Liu, H., Zhang, B., Cao, Q., et al. (2018c). Arabidopsis PCaP2 functions as a linker between ABA and SA signals in plant water deficit tolerance. Front. Plant Sci. 9:578. doi: 10.3389/fpls.2018.00578

Wu, J., Ichihashi, Y., Suzuki, T., Shibata, A., Shirasu, K., Yamaguchi, N., et al. (2019a). Abscisic acid-dependent histone demethylation during postgermination growth arrest in Arabidopsis. Plant Cell Environ. 42, 21982214. doi: 10.1111 pce. 13547

Wu, J., Yamaguchi, N., and Ito, T. (2019b). Histone demethylases control root elongation in response to stress-signaling hormone abscisic acid. Plant Signal. Behav. 14:1604019. doi: 10.1080/15592324.2019.1604019

Wu, M. F., Sang, Y., Bezhani, S., Yamaguchi, N., Han, S. K., Li, Z., et al. (2012). SWI2/SNF2 chromatin remodeling ATPases overcome polycomb repression and control floral organ identity with the LEAFY and SEPALLATA3 transcription factors. Proc. Natl. Acad. Sci. U.S.A. 109, 3576-3581. doi: 10.1073/ pnas.1113409109

Yamaguchi, N., Matsubara, S., Yoshimizu, K., Seki, M., Hamada, K., Kamitani, M., et al. (2020). Removal of repressive histone marks creates epigenetic memory of recurring heat in Arabidopsis. bioRxiv [Preprint] doi: 10.1101/2020.05.10. 086611

Yamaguchi, N., Winter, C. M., Wu, M. F., Kwon, C. S., William, D. A., and Wagner, D. (2014). PROTOCOLS: chromatin immunoprecipitation from Arabidopsis tissues. Arabidopsis Book 12:e0170. doi: 10.1199/tab. 0170

Yamaguchi-Shinozaki, K., and Shinozaki, K. (2006). Transcriptional regulatory networks in cellular responses and tolerance to dehydration and cold stresses. Annu. Rev. Plant Biol. 57, 781-803. doi: 10.1146/annurev.arplant.57.032905. 105444

Yamane, K., Toumazou, C., Tsukada, Y., Erdjument-Bromage, H., Tempst, P., Wong, J., et al. (2006). JHDM2A, a JmjC-containing H3K9 demethylase, facilitates transcription activation by androgen receptor. Cell 125, 483-495. doi: 10.1016/j.cell.2006.03.027

Yang, X., Bai, Y., Shang, J., Xin, R., and Tang, W. (2016). The antagonistic regulation of abscisic acid-inhibited root growth by brassinosteroids is partially mediated via direct suppression of ABSCISIC ACID INSENSITIVE 5 expression by BRASSINAZOLE RESISTANT 1. Plant Cell Environ. 39, 1994-2003. doi: 10. $1111 /$ pce. 12763

Yin, Y., Wang, Z. Y., Mora-Garcia, S., Li, J., Yoshida, S., Asami, T., et al. (2002). BES1 accumulates in the nucleus in response to brassinosteroids to regulate gene expression and promote stem elongation. Cell 109, 181-191.

Yoshida, T., Fujita, Y., Sayama, H., Kidokoro, S., Maruyama, K., Mizoi, J., et al. (2010). AREB1, AREB2, and ABF3 are master transcription factors that cooperatively regulate ABRE-dependent ABA signaling involved in drought stress tolerance and require ABA for full activation. Plant J. 61, 672-685. doi: 10.1111/j.1365-313X.2009.04092.x

Yu, X., Li, L., Li, L., Guo, M., Chory, J., and Yin, Y. (2008). Modulation of brassinosteroid-regulated gene expression by Jumonji domain-containing proteins ELF6 and REF6 in Arabidopsis. Proc. Natl. Acad. Sci. U.S.A. 105, 7618-7623. doi: 10.1073/pnas.0802254105

Zhang, X., Clarenz, O., Cokus, S., Bernatavichute, Y. V., Pellegrini, M., Goodrich, J., et al. (2007). Whole-genome analysis of histone $\mathrm{H} 3$ lysine 27 trimethylation in Arabidopsis. PLoS Biol. 5:e129. doi: 10.1371/journal.pbio.0050129

Zhang, X., Zhou, L., Qin, Y., Chen, Y., Liu, X., Wang, M., et al. (2018). A temperature-sensitive misfolded bri1-301 receptor requires its kinase activity to promote growth. Plant Physiol. 178, 1704-1719. doi: 10.1104/pp.18.00452

Zheng, S., Hu, H., Ren, H., Yang, Z., Qiu, Q., Qi, W., et al. (2019). The Arabidopsis H3K27me3 demethylase JUMONJI 13 is a temperature and photoperiod dependent flowering repressor. Nat. Commun. 10:1303. doi: 10.1038/s41467019-09310-x

Zhu, L., Zhang, Y., Kang, E., Xu, Q., Wang, M., Rui, Y., et al. (2013). MAP18 regulates the direction of pollen tube growth in Arabidopsis by modulating F-actin organization. Plant Cell 25, 851-867. doi: 10.1105/tpc.113.110528

Conflict of Interest: The authors declare that the research was conducted in the absence of any commercial or financial relationships that could be construed as a potential conflict of interest.

Copyright (C) $2020 \mathrm{Wu}$, Yan, Zhang, Zhou, Yamaguchi and Ito. This is an open-access article distributed under the terms of the Creative Commons Attribution License (CC BY). The use, distribution or reproduction in other forums is permitted, provided the original author(s) and the copyright owner(s) are credited and that the original publication in this journal is cited, in accordance with accepted academic practice. No use, distribution or reproduction is permitted which does not comply with these terms. 